\title{
Finite size effects on instabilities of discrete breathers
}

\author{
J.L. Marín ${ }^{\mathrm{a}, *}$, S. Aubry ${ }^{\mathrm{b}, 1}$ \\ a Departamento de Física de la Materia Condensada, Instituto de Ciencia de Materiales de Aragón, \\ Universidad de Zaragoza, 50009 Zaragoza, Spain \\ b Laboratoire Léon Brillouin (CEA-CNRS), CE Saclay, 91191-Gif-sur-Yvette, Cedex, France
}

Received 30 May 1997; received in revised form 10 October 1997

\begin{abstract}
When the two arcs of the continuous phonon spectrum of the Floquet matrix of a discrete breather overlap on the unit circle, the breather solution in the infinite lattice might be stable while the corresponding solutions in finite systems appear to be unstable. More precisely, when the model parameters vary, the breather in the finite system exhibits a large number of collisions between the Floquet eigenvalues belonging to the phonon spectrum. These collisions correspond to complex cascades of instability thresholds followed near after by re-entrant stability thresholds.

We interpret this complex structure on the basis of the band analysis of the matrix of the second variation of the action. Then we can predict that in the limit of an infinite system the number of instability and stability thresholds in the cascade diverges, but simultaneously the maximum amplitude of the instabilities vanishes, so that the breather in the infinite system recovers its linear stability (as long as all its other localized modes remain stable). This is the situation which is required in Cretegny et al. [Physica D 119 (1998) 73-87] for having inelastic phonon scattering with two channels.

We also analyze the size effects when a Floquet eigenvalue associated with a localized mode collides with the Floquet continuous phonon spectrum with different Krein signature. In contrast to the previous case, the infinite system is unstable after the collision. (C) 1998 Elsevier Science B.V.
\end{abstract}

\section{Introduction}

Unlike the exceptional solutions of some integrable models, discrete breathers are time-periodic and spatially localized solutions which are robust to perturbations and may exist in many anharmonic models. A series of works initiated by the pioneering work of Sievers and Takeno [2] have demonstrated that they are ubiquitous in highly anharmonic systems (for a review see [3]). These results were completed later by a

\footnotetext{
* Corresponding author. E-mail: marin@wanda.unizar.es.

${ }^{1}$ E-mail: aubry@bali.saclay.cea.fr.
}

mathematical proof of existence valid for large classes of coupled anharmonic oscillators [4], and a corollary to this theorem was to provide a highly accurate numerical method for calculating any of these solutions by continuation from their anticontinuous limit [5]. Then, it becomes possible to perform an accurate stability analysis of the obtained solutions through a standard Floquet analysis.

Our purpose here is to focus on one aspect of the stability of breathers which involves the size of the system. Indeed, one of the conclusions of [4] is that discrete breathers exist for the infinite system as well as for finite systems. For numerical calculations, we 
consider here finite systems with the standard periodic boundary conditions, although we could study as well different boundary conditions.

Let us now illustrate our results by considering as an example the well-known 1D Klein-Gordon (KG) model which consists of anharmonic oscillators with mass unity and harmonic nearest neighbor coupling with constant $C$. Its Hamiltonian is

$\mathbf{H}=\sum_{n}\left(\frac{p_{n}^{2}}{2}+V\left(u_{n}\right)+\frac{C}{2}\left(u_{n+1}-u_{n}\right)^{2}\right)$

We consider here the cubic potential $V(x)=x^{2} / 2-$ $x^{3} / 3$, which has its linear limit frequency normalized to $\omega_{0}=1$. The single-site breather solution of this equation with period $t_{\mathrm{b}}$ and frequency $\omega_{\mathrm{b}}=2 \pi / t_{\mathrm{b}}$ is a time-periodic solution $\left\{u_{n}(t)\right\}$ of the dynamical equations

$\ddot{u}_{n}+V^{\prime}\left(u_{n}\right)-C\left(u_{n+1}+u_{n-1}-2 u_{n}\right)=0$,

which is obtained by continuation from $C=0$ (anticontinuous limit) of a solution $\left\{v_{n}(t)\right\}$ in which $v_{n}(t) \equiv 0$ for $n \neq 0$ and $v_{0}(t)$ is a periodic solution (with period $t_{\mathrm{b}}$ ) of the single uncoupled oscillator with potential $V(x)$.

The linear stability of the breather is determined by definition by the linear equation:

$$
\begin{aligned}
& \ddot{\epsilon}_{n}+V^{\prime \prime}\left(u_{n}(t)\right) \epsilon_{n} \\
& \quad-C\left(\epsilon_{n+1}+\epsilon_{n-1}-2 \epsilon_{n}\right)=0 .
\end{aligned}
$$

These form a set of coupled linear differential equations with periodic coefficients, analogous to a problem of coupled Schrödinger equations for electrons in a periodic potential $V^{\prime \prime}\left(u_{n}(t)\right)$, if we make the change $\Psi_{n}(x) \leftrightarrow \epsilon_{n}(t)$. Note that this equation always has the trivial solution $\epsilon_{n}(t)=\dot{u}_{n}(t)$, the "phase mode" corresponding to time translation.

For a system of size $N$, the integration of Eqs. (3) over a period $t_{\mathrm{b}}$ of each of the $2 N$ vectors $\left\{\epsilon_{n}(0), \dot{\epsilon}_{n}(0)\right\}$ forming some basis of the tangent space yields the Floquet matrix F. This $2 N \times 2 N$ matrix represents a general solution of Eqs. (3), since it is the mapping at a period relating linearly $\left\{\epsilon_{n}\left(t_{b}\right), \dot{\epsilon}_{n}\left(t_{b}\right)\right\}=\mathbf{F}\left\{\epsilon_{n}(0), \dot{\epsilon}_{n}(0)\right\}$.
Since the system (3) is Hamiltonian, $\mathbf{F}$ is symplectic, which (together with the fact that the system is real) implies that if $\lambda$ is an eigenvalue $\lambda^{*}, 1 / \lambda$, and $1 / \lambda^{*}$ are also eigenvalues. The linear stability of the breather requires that the $N$ pairs of eigenvalues $\mathrm{e}^{ \pm \mathrm{i} \theta_{v}}$ of the Floquet matrix are on the unit circle. The phase mode is spatially localized and corresponds to a degenerate pair of eigenvalues at $+1(\theta=0)$. Near the anticontinuous limit, the rest of the eigenmodes are close to $\pm \theta_{0}= \pm 2 \pi \omega_{0} / \omega_{\mathrm{b}}$ modulo $2 \pi$. In [4] singlesite breathers are proven to be linearly stable near this limit, i.e., all the eigenvalues of the Floquet matrix lie on the unit circle.

When following the breather family in the model parameters from $C=0$, the eigenvalues of the Floquet matrix vary continuously. When an isolated eigenvalue $\mathrm{e}^{\mathrm{i} \theta}$ and its complex conjugate $\mathrm{e}^{-\mathrm{i} \theta}$ collide either at $\theta=0$ or at $\theta=\pi$, the generic behavior is for them to perform an excursion out of the unit circle (i.e., the breather becomes unstable). Also, when two isolated eigenvalues with different Krein signatures [6] collide at any angle, an instability is expected to occur ("Krein crunch"), whereby a quadruplet of eigenvalues gets out of the unit circle. Indeed, many kinds of instabilities were found by the numerical investigation of the Floquet eigenvalues $[7,8]$, but it was also found that many instabilities were due to size effects and thus are not relevant for very large (or infinite) systems. These size effects originate from the discretization of the phonon spectrum of the Floquet matrix of the infinite system.

It is the purpose of this paper to discuss this size effect. For the understanding of the following discussion the reader will need to refer to the section in [9] dealing with a new approach based on a band analysis of the spectrum of the matrix of the second variation of the action, which recovers and extends the prediction of the Krein theory for analyzing the stability of breathers.

However, we can provide some physical insight into the problem, before we move on to more rigorous details. In principle it is natural to expect phenomena of parametric resonance, i.e., Floquet eigenmodes out of the unit circle. For our discussion we consider only breathers originated from a finite 
number of sites, and we may take the single-site breather as a working example, without loss of generality. Then the stability problem (3) can be viewed as a system of linear, coupled parametric oscillators. The time-dependent term $V^{\prime \prime}\left(u_{n}(t)\right)$ is to be viewed here as an external periodic excitation which goes exponentially fast to 1 , due to exponential localization of discrete breathers. Then, modes associated with strong instabilities should be located for their essential part in the region of the breather (i.e., be also localized). There may also exist more extended and weaker instabilities, but we will show that they strictly disappear when the system size diverges. We may anticipate the line of reasoning: in infinite systems, resonances which involve extended modes cannot exist because, in simple terms, developing an instability far away from the breather would also imply (because the breather just introduces a negligible perturbation on the spectrum of the extended system) that the ground state is also unstable, in contradiction with the assumptions.

At this point it is necessary to clarify that we are adopting the customary definition of linear stability in physical problems: instability implies exponential growth with time. However, there are situations where the linear stability analysis yields modes which are neither exponentially growing nor of Bloch type, but algebraically growing. In this paper we set out to prove the non-existence of extended, exponentially unstable modes in the limit of the infinite lattice, under rather generic assumptions. The question of possible algebraic growth of these extended modes (leading thus to breather radiation) is explored in [9], but needs further investigation.

Finally, it is worth mentioning the possible relevance of these finite size effects in the kind of phenomena that was observed in one of the pioneering works on discrete breathers, by Campbell and Peyrard [10]. These authors calculated numerically discrete breathers in a $\phi^{4}$ potential, and the Floquet analysis showed an intricate structure of stable and unstable zones. Although it is not clear yet whether or not those solutions are the same as the ones obtained "cleanly" from the uncoupled limit, it is very likely that similar finite size effects are at work there [11].

\section{The nature of the continuous spectrum}

In the limit of an infinite system $N=\infty$, the spectrum of $\mathbf{F}$ consists of a continuous part associated with spatially extended eigenvectors and a discrete part consisting of eigenvalues associated with spatially exponentially localized eigenvectors. The continuous part of the spectrum of $\mathbf{F}$ is simply the continuous spectrum which would be obtained for the system without breather, i.e., when $V^{\prime \prime}\left(u_{n}(t)\right)=V^{\prime \prime}(0) \equiv 1$ for all $n$. In what follows we give a few arguments for this assertion.

Using the fact that the limit (in the weak topology) of the sequence of spatial translations of the Floquet matrix $\mathbf{F}$ of the system with breather is the Floquet matrix $\mathbf{F}_{0}$ of the system without breather, one proves easily that the whole spectrum of the Floquet matrix $\mathbf{F}_{0}$ is included in the spectrum of the initial Floquet matrix F. The eigenvectors $\left\{\epsilon_{n}(q), \dot{\epsilon}_{n}(q)\right\}=\left\{\mathrm{e}^{\mathrm{i} q n}, \mathrm{i} \omega(q) \mathrm{e}^{\mathrm{i} q n}\right\}$ of $\mathbf{F}_{0}$, which are invariant under any spatial translation, just correspond to plane waves and depend on the continuous wave vector $q$. Their corresponding eigenvalues are $\mathrm{e}^{\mathrm{i} \omega(q) t_{\mathrm{b}}}$, where $\omega(q)= \pm \sqrt{1+4 C \sin ^{2}(q / 2)}$ and $t_{b}=2 \pi / \omega_{b}$. These are just the normal modes of the linear lattice, and thus we loosely refer to them as the phonon spectrum or phonon band. ${ }^{2}$

Reciprocally, we can consider a bounded and extended (pseudo) eigenvector $\mathbf{X}_{0}=\left\{\epsilon_{n}(0), \dot{\epsilon}_{n}(0)\right\}=$ $\left\{X_{n}\right\}$ of $\mathbf{F}$. Its corresponding eigenvalue $\lambda$ is in the continuous spectrum of the Floquet matrix $\mathbf{F}$. Since by hypothesis, $X_{n}$ does not go to zero for $|n| \rightarrow \pm \infty$, the sequence of translated vectors $\mathbf{X}_{p}=\left\{\epsilon_{n+p}(0), \dot{\epsilon}_{n+p}(0)\right\}$ has accumulation points for the weak topology $\mathbf{X}_{\infty}=\left\{\eta_{n}(0), \dot{\eta}_{n}(0)\right\} \neq \mathbf{0}$. This can be achieved by constructing recursively an infinite monotone subsequence $p_{i}$ such that for any $n$, we have $\lim _{i \rightarrow \infty} \epsilon_{n+p_{i}}(0)=\eta_{n}(0)$ and $\lim _{i \rightarrow \infty} \dot{\epsilon}_{n+p_{i}}(0)=\dot{\eta}_{n}(0)$ (the limits do not have to be uniform). These vectors $\mathbf{X}_{\infty}$ are eigenvectors of the limit of the Floquet matrices translated by $p_{i}$, for

\footnotetext{
${ }^{2}$ Unfortunately, there is a clash in terminology when we deal below with the "bands" structure of the Hill's equation, Eq. (5). Therefore we prefer the term "spectrum" when applied to phonon modes.
} 
the same eigenvalue $\lambda$. The limit vector at the accumulation point is an eigenvector of the limit Floquet matrix $\mathbf{F}_{0}$, i.e., a plane wave.

Therefore, the continuous part of the spectrum of the Floquet matrix $\mathbf{F}$ is on the unit circle and essentially consists of the two arcs defined by

$$
\begin{aligned}
\theta_{0} & \equiv \frac{2 \pi}{\omega_{\mathrm{b}}}-2 \pi p \leq \theta \leq \frac{2 \pi \sqrt{1+4 C}}{\omega_{\mathrm{b}}}-2 \pi p \\
& \equiv \theta_{\mathrm{top}}
\end{aligned}
$$

and the symmetric arc corresponding to $-\theta$. The offsets $2 \pi p$ are written here explicitly, in order to bring $\left[\theta_{0}, \theta_{\text {top }}\right]$ into the range $[0,2 \pi]$. The existence of spatially localized breathers generally requires that its harmonic frequencies $p \omega_{b}$ are not in the phonon band for any integer $p$. This property avoids the decay of the breather energy by radiation in the phonon band. ${ }^{3}$ As a result, the two symmetric arcs do not contain the point $\theta=0$.

When $C$ increases from zero, the width of these two $\operatorname{arcs} \theta_{\text {top }}-\theta_{0}=2 \pi(\sqrt{1+4 C}-1) / \omega_{\mathrm{b}}$ increases, and there may exist situations where the leading edges of the arc $\pm \theta_{\text {top }}$ reach $\theta=\pi$. To be precise, this happens when $\omega_{\mathrm{b}} \in(2, \infty)$ for hard potentials $V$, and when $\omega_{\mathrm{b}} \in(2 / 3,1), \omega_{\mathrm{b}} \in(2 / 5,1 / 2), \omega_{\mathrm{b}} \in(2 / 7,1 / 3)$, etc. for soft potentials. In all these cases the two arcs start to overlap while the breather still exists. When this situation occurs, there are, in some sense, infinitely many eigenvalues of the continuous spectrum (with different Krein signature) which collide. A naive extension of the Krein theory for infinite systems applied to such eigenvalues collisions would suggest that breather instabilities could be generated.

Actually, no breather instability can result from an extended mode (if the ground-state of the model is stable, of course) because as shown above, the corresponding eigenvalue of the Floquet matrix $\mathbf{F}$ must belong to one of the two arcs (4) on the unit circle and has modulus one. In our case, the eigenvectors corresponding to the colliding eigenvalues of the two

\footnotetext{
${ }^{3}$ Actually, there are models with exact breather solutions and harmonics in the phonon spectrum (e.g., sine-Gordon), but it has been shown that the absence of radiation is exceptional due to the integrability properties, and that this exact solution disappears under most Hamiltonian perturbations.
}

overlapping arcs are extended and therefore asymptotic at infinity to different plane waves, which cannot generate by a combination on a non-extended mode. We see how, in this case, the validity of the extension of the Krein theory to the collisions between eigenvalues of the continuous spectrum is questionable.

The situation is different for finite systems, where the continuous spectrum of the Floquet matrix is replaced by a finite, discrete set of eigenvalues. In that situation, the standard Krein theory fully applies. Indeed, finite systems show the appearance of many small instabilities due to collisions between the discrete eigenvalues (with different Krein signatures) which take place on what otherwise would be the continuous spectrum. A more careful analysis will show here that when the size of the system is increased, the magnitude of these instabilities weakens uniformly, while at the same time, due to an increasing density of colliding eigenvalues, their number increases. Thus when the size of the system is infinite, we should expect no more instabilities caused by the two arcs of the continuous spectrum overlapping.

\section{Numerical results}

We illustrate our interpretation for the single breather of the cubic KG equation (2) with frequency $\omega_{\mathrm{b}}=0.75$. In that case, the two arcs of the continuous spectrum of the Floquet matrix are determined by $\theta_{0}=2 \pi / 3$ and $\theta_{\text {top }}=2 \pi(4 / 3 \sqrt{1+4 C}-1)$. These two arcs overlap when $\theta_{\text {top }} \geq \pi$, i.e., when $C>17 / 256 \approx 0.066406$. Fig. 1 shows the variation of the arguments $\theta_{v}$ of the eigenvalues of the Floquet matrix on the unit circle in terms of the continuation parameter $C$, for different system sizes. There are two discrete but dense clusters of eigenvalues covering uniformly the two arcs of the continuous spectrum, which become denser as the size of the system $N$ increases. Beyond some values of $C$, some eigenvalues may leave the arcs and become isolated from the continuous spectrum, a phenomenon better spotted when the system size is sufficiently large. They are associated with spatially localized eigenmodes [12]. In the figure we clearly appreciate the formation of at 

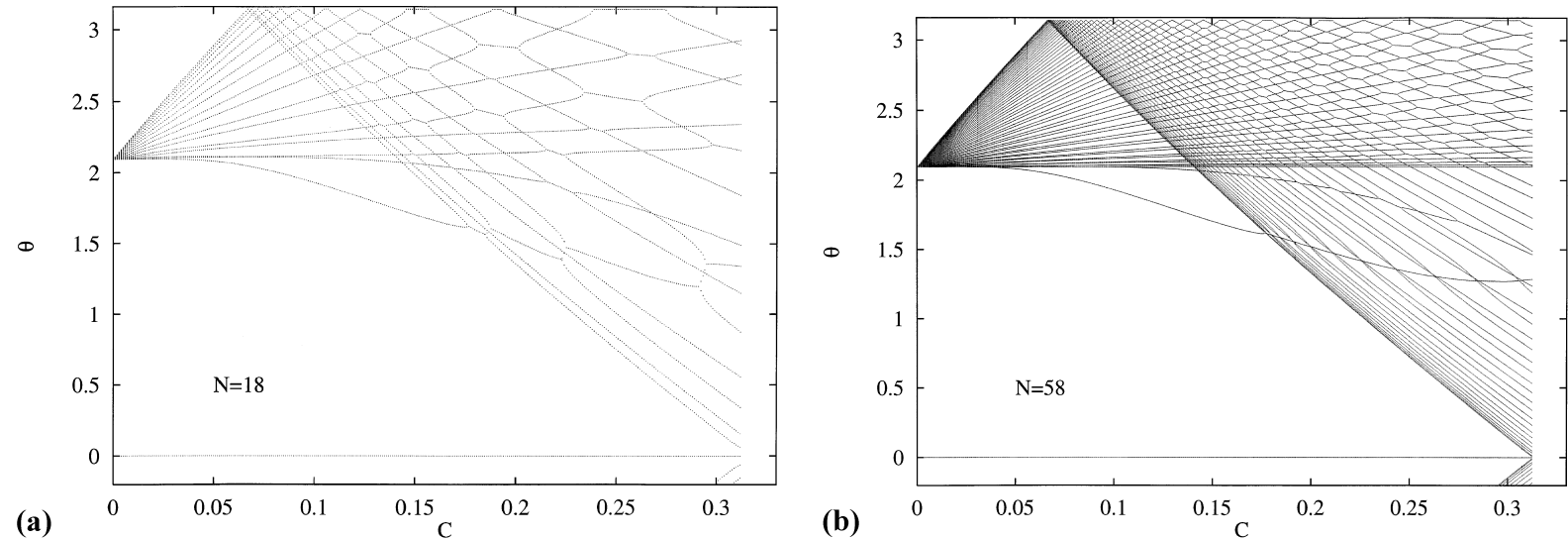

Fig. 1. Argument $\theta_{v}$ vs. $C$ of the eigenvalues $\lambda=\rho_{v}^{ \pm 1} \mathrm{e}^{ \pm \mathrm{i} \theta_{v}}$ of the Floquet matrix $\mathbf{F}$ for a single breather in the cubic KG model (1) at frequency $\omega_{\mathrm{b}}=0.75$ and for system sizes $N=18$ and $N=58$.
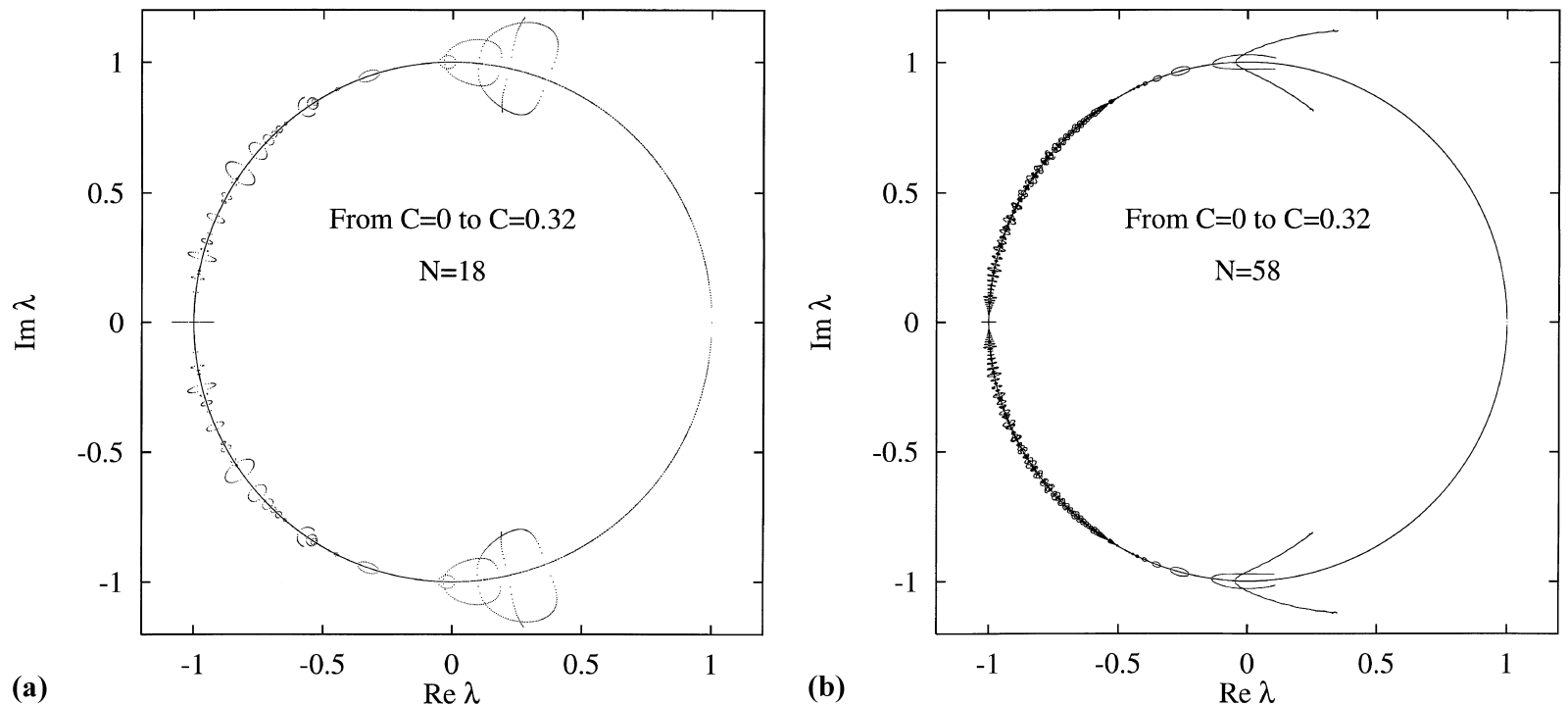

Fig. 2. Global trace of the eigenvalues of the Floquet matrix when $C$ varies from 0 to $\approx 0.32$, for the same breather and system sizes as in Fig. 1.

least two of such localized modes detaching from the phonon spectrum.

The single breather we consider has a spatial center of symmetry $u_{i}(t)=u_{-i}(t)$, therefore the eigenmodes of the Floquet matrix $\mathbf{F}$ are either spatially symmetric or antisymmetric. When a pair of eigenvalues $\mathrm{e}^{\mathrm{i} \theta_{1}}$ and $\mathrm{e}^{\mathrm{i} \theta_{2}}$ corresponding to eigenmodes with different spatial symmetry collide, they cross each other and no instability appears. On the other hand, when some pair of eigenvalues $\mathrm{e}^{\mathrm{i} \theta_{1}}$ and $\mathrm{e}^{\mathrm{i} \theta_{2}}$ corresponding to eigenmodes with the same spatial symmetry collide, the two eigenvalues get out of the unit circle. They take the form $\rho \mathrm{e}^{\mathrm{i} \theta}$ and $\rho^{-1} \mathrm{e}^{\mathrm{i} \theta}$ with the same argument $\theta$. This phenomena is clearly visible in the crossings of Fig. 1. We also observe that in most cases, shortly after such an instability threshold, each pair of eigenvalues returns on the unit circle after an inverse Krein crunch. The net result is the same as if 


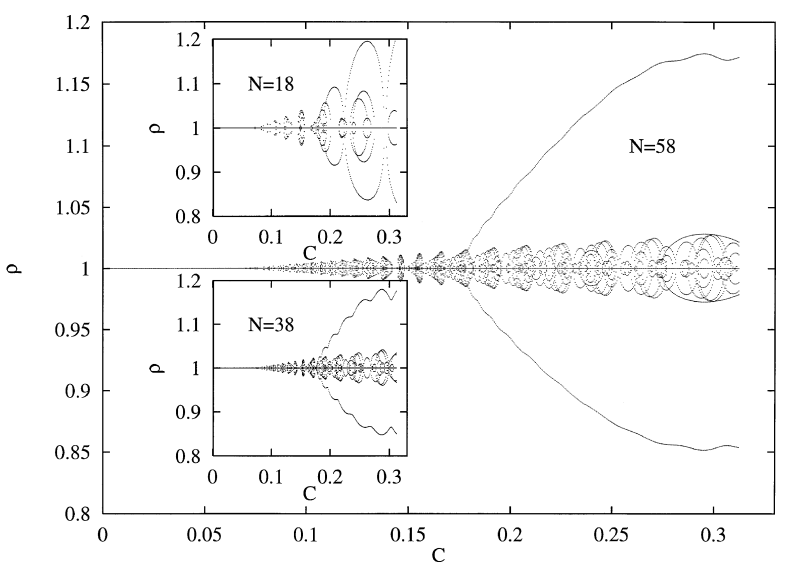

Fig. 3. Modulus of the eigenvalues of the Floquet matrix vs. $C$ for the same breather as Fig. 1, for a system size $N=58$ (insets: same for $N=18$ and $N=38$ ).

the two eigenvalues $\mathrm{e}^{\mathrm{i} \theta_{1}}$ and $\mathrm{e}^{\mathrm{i} \theta_{2}}$ would have crossed each other without interacting. There are also many eigenvalue collisions where the Krein crunches and their subsequent inverse Krein crunches are indistinguishable, depending on the precision of the numerics.

Fig. 2 shows the global trace of the eigenvalues of the Floquet matrix $\mathbf{F}$ on the complex plane, for the same breather as in Fig. 1, when $C$ varies from 0 to approximately 0.32 . Note that when $C=\omega_{\mathrm{b}}^{2}-1 / 4$, i.e., $C=5 / 16=0.3125$, the second harmonic of the breather enters the phonon spectrum and it is then continuable as a new type of extended nonlinear solution, the "phono-breather" [9], which is not studied here. The figures confirm how the intra-band instabilities reduce when the size of the system increases. However, there are cases in which we can also observe at least one instability which persists at larger sizes (although it necessarily involves localized modes, as we proved). We chose the cubic model because it offers such an example clearly. Fig. 3 plots the modulus of the eigenvalues in lattices of different sizes, making it more apparent. A calculation in successively bigger systems not only confirms the shrinking of instabilities due to extended modes, but uncovers the persistence of such instability. This takes place when a localized mode of the spectrum is reached by the phonon modes, which after having crossed with each other through $\theta=\pi$, have changed their Krein signature. We treat this specific case under the general band analysis technique in the next section.

\section{Interpretation in terms of the band analysis}

We confirm and interpret our study by a band analysis of the spectrum of the second variation of the action (see [9]). The basic idea of the method is to study an augmented problem in which the arguments of the Floquet eigenvalues appear as the intersection points of bands with an axis $E=0$. The existence of a bands structure implies topological constraints on the variation of the Floquet eigenvalues. For example the proof of the Krein theory is straightforward in this representation. It can also help in the interpretation of puzzling numerical results [13]. In addition, those who are familiar with band theory, can recognize features which are usual for band interactions. The band structure we consider is generated by the eigenequation

$$
\begin{aligned}
& \ddot{\epsilon}_{n}+V^{\prime \prime}\left(u_{n}(t)\right) \epsilon_{n} \\
& \quad-C\left(\epsilon_{n+1}+\epsilon_{n-1}-2 \epsilon_{n}\right)=E \epsilon_{n} .
\end{aligned}
$$

Since $V^{\prime \prime}\left(u_{n}(t)\right)$ is time-periodic with period $t_{\mathrm{b}}$, the eigensolutions of Eq. (5) fulfill the Bloch condition

$\epsilon_{n}\left(t+t_{\mathrm{b}}\right)=\epsilon_{n}(t) \mathrm{e}^{\mathrm{i} \theta}$.

This theorem implies that each eigenvalue depends on $\theta$ as a symmetric $2 \pi$-periodic smooth functions $E(\theta)$ called bands. The terminology comes obviously from condensed matter physics [14], in the context of electrons in periodic potentials, and from the equivalent problem of the stability zones of the Hill's equation [15].

For a finite system, the whole spectrum of Eq. (5) can be represented by a discrete (but infinite) set of bands $E_{v}(\theta)$. For an infinite system, this band structure consists of some discrete isolated bands (corresponding to spatially localized eigenmodes) and a "continuous part" (spatially extended eigenmodes) where the bands depend continuously on an extra parameter (e.g., a spatial wave vector $q$ ). According to [9], the arguments $\theta_{v}$ of the eigenvalues $\mathrm{e}^{\mathrm{i} \theta_{v}}$ of the Floquet matrix $\mathbf{F}$ which are on the unit circle are given by the 

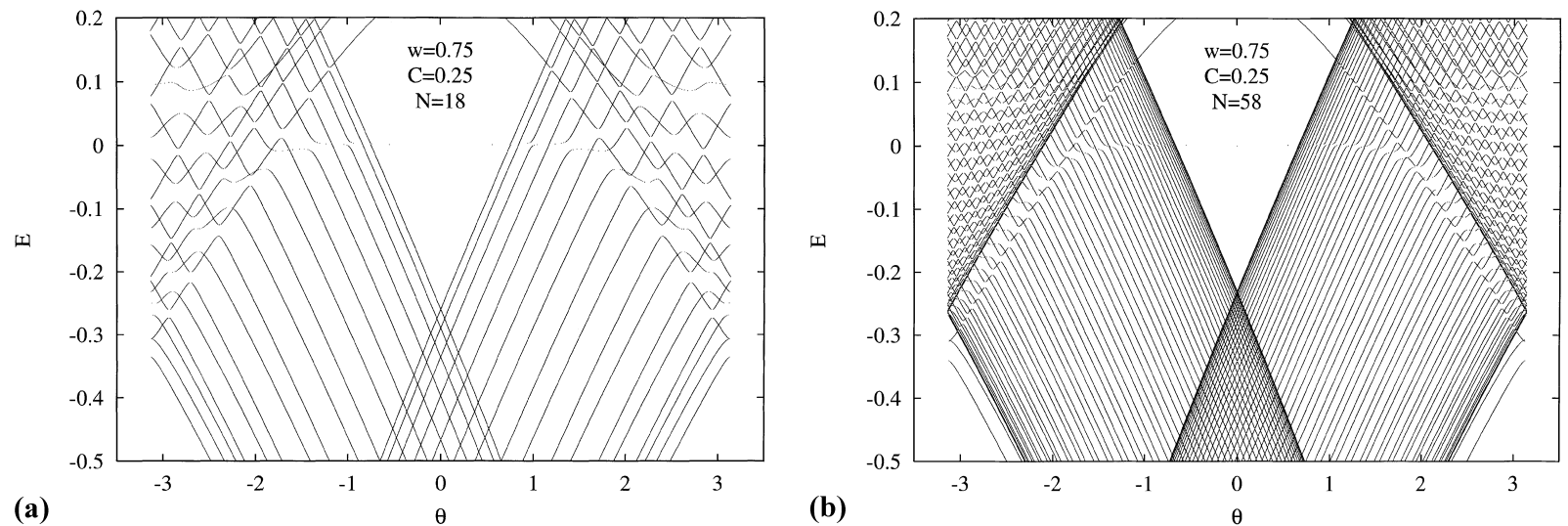

Fig. 4. Band structure of Eq. (5) for the single breather in the cubic KG model equation (1) at $C=0.25$ and $\omega_{\mathrm{b}}=0.75$, for two system sizes: $N=18$ and $N=58$. The spatially symmetric and antisymmetric bands alternate and do not interact.

intersection of the bands $v$ with the line $E=0$. The associated Krein signature is the opposite of the slope $\mathrm{d} E_{v} / \mathrm{d} \theta$ at such intersection in the interval $0<\theta<\pi$.

The part of the band structure in a neighborhood of $E=0$ has been numerically calculated for the single breather of the cubic KG chain for several sizes (see Fig. 4). For a finite system with size $N$, the band structure is discrete and there are at most $N$ bands intersecting $E=0$, since the Floquet matrix has at most $N$ pairs of eigenvalues on the unit circle. If the breather is linearly stable there are precisely $N$ bands intersecting $E=0$ (including the band which is tangent at $\theta=0$ and yields the phase mode).

The global knowledge of the band structure in the vicinity of $E=0$ allows one to have a better understanding of the collisions between eigenvalues and the instabilities which may or may not result. Particularly, the cascade of re-entrant instabilities observed in Figs. 1-3 can be interpreted through a rather standard band analysis familiar to solid state physicists.

The band structure can easily be calculated both for the infinite and the finite system without breather. For the infinite system, the eigensolutions of Eq. (5) are $\epsilon_{n}(t)=\mathrm{e}^{\mathrm{i}(q n-\omega(q, E) t)}$ with $\omega^{2}(q, E)=$ $1+4 C \sin ^{2}(q / 2)-E$. These solutions fulfill Eqs. (6) with $\theta=\omega(q, E) t_{\mathrm{b}}+2 \pi p$ in the interval $[-\pi, \pi]$ for some integer $p$. Then, we get a particular band in the continuum of bands by choosing the spatial wave vector $q$ (and the folding index $p$ ):
$E(\theta, q)=1+4 C \sin ^{2} \frac{q}{2}-\frac{\omega_{\mathrm{b}}^{2}}{4 \pi^{2}}(\theta+2 \pi p)^{2}$.

For a finite system with size $N$, the spatial wave vector $q$ is discretized as $q_{v}=2 \pi v / N$ with $v$ an integer. When there are no breathers in the system, the bands (7) are doubly degenerated for $q$ and $-q$. In addition, since they are obtained by the folding in the interval $[-\pi, \pi]$ of a bunch of parallel and infinite curves, they intersect many other bands (with a slope of opposite sign) in a vicinity of their crossings with the vertical axes $\theta=0$ and $\theta= \pm \pi$. At these intersections, there is also degeneracy in the eigenvalues of $\mathbf{F}(E)$.

When the breather is introduced, these degeneracies will be raised. Let us consider first regions in $E$ and $\theta$ where the bands are parallel and where there are no band intersections, i.e., only a degeneracy between the solutions with wave vector $+q$ and $-q$, but no degeneracy with other solutions with different wave vector. In our case, the single breather is spatially symmetric, so that raising this degeneracy yields a spatially symmetric and a spatially antisymmetric eigensolution. The effect appears as a slight separation of the associated bands, as can be appreciated in Fig. 4(b) in the regions where they do not intersect with other bands. It is worth mentioning here that this "splitting" of the bands (the ones associated with extended phonon modes) is directly related to the scattering properties of breathers with respect to plane waves, as explained in [1]. 
Let us now consider the more complex regions in $E$ and $\theta$ where there are transversal intersections between the bands. When two bands with the same spatial symmetry intersect transversally (or, equivalently, have different Krein signature in the vicinity of the intersection [9]), the degeneracy between the two bands is raised locally, and a gap opens up. On the contrary, if the two intersecting bands have the same slope sign (i.e., Krein signature), no gap appears, even if they have the same spatial symmetry. These interactions can be understood within a standard perturbation approach of band theory (e.g., Peierls instability in $1 \mathrm{D}$ conductors [14]), where the breather creates the perturbative potential. Krein theory is thus recovered through standard techniques of solid state physics.

The amplitude of the gap in $E$ is at the lowest order equal to the modulus of the overlap of the symmetric (or antisymmetric) normalized eigenmodes of the system, Eq. (5). The non-vanishing contribution of this overlap is essentially restricted to the finite region of the space where there is a perturbation, i.e., where the breather is located. If both eigenmodes are spatially extended, and the breather size is finite and well defined, this overlap is of the order of the inverse size $1 / N$ of the system. As a result, the gap width at the intersections is expected to be of the same order of magnitude $1 / N$, and thus goes to zero when the system size diverges. We recover again the same result we obtained in Section 2.

These features are compatible with the observed behavior at different sizes shown in Fig. 4. For a reasonably smooth model, the extended Floquet matrix $\mathbf{F}(E)$ obtained from a one-period integration of Eq. (5) for $E \neq 0$ has a bounded derivative with respect to $E$. Thus, the maximum variation of each eigenvalue of $\mathbf{F}(E)$ in its instability gaps is also of order $1 / N$, and consequently its maximum distance from the unit circle remains of order $1 / N$.

However all these arguments may not apply when one of the colliding bands has spatially localized associated eigenvectors. Intersections between two of such localized bands having different signatures (and same spatial symmetry) are expected to develop gaps in such a way that both the width of the gap and the magnitude of the instability approach rapidly a constant (non- zero) value as the lattice size grows. But the question of intersections between a band of localized character and the continuum of bands of extended character is more complicated, and is devoted an interpretation in the next section.

\section{Localized modes colliding with the continuous spectrum}

Beside the band overlap phenomena, it can be seen on Figs. 2 and 3 that there is clearly an instability by a spatially localized mode which persists for large sizes. Actually, a more careful analysis reveals a second instability. These two instabilities are due to the fact that two Floquet eigenvalues on the unit circle associated with spatially symmetric and antisymmetric localized modes collide with the part of the continuous phonon spectrum of the Floquet matrix which has different Krein signature. After the collision, the manifestation of each mode crossing the continuous spectrum consists of "wiggles" on the discrete bands of finite systems (only those with the same spatial symmetry), which we will interpret here.

Those wiggles associated with the antisymmetric mode are clearly visible in Fig. 4, while those associated with the symmetric mode are more elusive, but clearly revealed by the magnification of Fig. 5. These

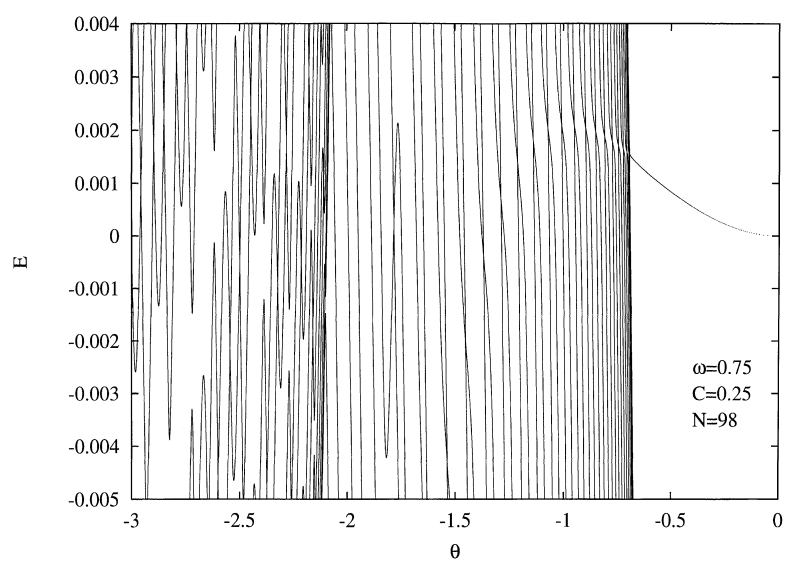

Fig. 5. Magnification of the band structure of Fig. 4 close to the axis $E=0$, but for a larger size $N=98$. The band associated with the phase mode (which has a poor resolution and is hardly visible on Fig. 4) collides with the phonon spectrum. 
wiggles vaguely draw the continuation of the band curves of the localized mode through the phonon spectrum. But the relevant feature for the breather stability is that (at sufficiently large sizes $N$ ) these wiggles conceal a real instability gap. In other words, there is no localized band present there.

Although there are exceptions with some special models, in general, a localized mode at frequency $\omega_{1}$ cannot strictly penetrate the phonon spectrum and remain localized, for the following trivial reason: it has generally non-vanishing and exponentially localized components at all the frequencies $\omega_{\mathrm{l}}+p \omega_{\mathrm{b}}$ for $p$ an integer. If one of these frequencies penetrates the phonon spectrum, the corresponding component should be spatially extended, which contradicts the assumption that the eigenmode is localized. ${ }^{4}$ Therefore two situations have to be considered when an isolated eigenvalue collides with the continuous phonon spectrum, depending on the Krein signature of the colliding eigenvalues.

When the Krein signature of the localized mode is the same as those of the bands in the phonon spectrum, this localized mode extends and joins the eigenstates of the phonon spectrum. No breather instability occurs. Examples can be found in [1], where localized modes were found to detach from the continuous phonon spectrum and then go back towards it. However, the trace of the penetration of this mode inside the extended bands can persist as a sharp positive variation of their phase shift (the wiggles) with amplitude $2 \pi$ around some wave vector $q_{1}$ (resonance), the position of which varies with the model parameters. ${ }^{5}$ See [1] for the details.

On the other hand, when the Krein signatures are different, a collision between the continuous phonon spectrum and a localized eigenmode generally produces a real instability for the infinite system, in sharp

\footnotetext{
${ }^{4}$ Of course, this argument should be amended when there are symmetries in the model which vanish systematically some of these harmonics. For example, the even harmonics would vanish in a KG chain with a symmetric quartic potential $V(x)=$ $V(-x)$, instead of the cubic one considered here.

${ }^{5}$ In [1] it is shown that the variation of the phase shift through the band is related to the number of localized modes outside the band.
}

contrast with the previously studied case of collision of two continuous arcs of extended phonon modes. This instability is also quite sensitive to size effects. In some cases this instability is very weak and the corresponding eigenmode is so weakly localized that it cannot be directly measured because the system size which would be required for that goes beyond our numerical capabilities (see an example in [1] for the "filtering breather").

The existence of this type of instability at large size can be confirmed on the base of the following arguments. In a finite system one can observe how the localized band develops a structure of gaps as it tries to cross the extended bands. The situation is depicted schematically in Fig. 6(a). For every particular band of the extended phonon spectrum, this results in a local sharp variation (phase-shift) of $\theta$, with the peculiarity that the band may become non-monotonous at that point. This is where the size effects appear. There are many pairs of re-entrant instability and stability thresholds associated with the gaps. For simplicity, the schemes of Fig. 6 do not take into account any spatial symmetry of the breather and assume that there is a gap opening at each intersection.

For the same but large enough system, the discrete phonon band determinations become univaluated, and the breather becomes unstable as soon as the localized mode collides with the first phonon band on one side. There is no stability re-entrance until the localized mode exits by one or the other side of the set of discrete bands corresponding to the continuous spectrum of the infinite system. This is depicted in Fig. 6(b).

Incidentally, we should mention that the minimum system size beyond which the discrete bands recover their monotonicity can be quite large, up to $N \simeq 1000$ (as it can be predicted by accurate calculations of the phase shift, using an alternative and more efficient technique which requires only relatively small systems; see [1]). Here we chose the cubic KG model because it shows the phenomenon at relatively small lattice sizes. On the other hand, we remind the reader that the whole problem of localized vs. extended modes in the spectrum of breathers is intimately related to the transmission properties of breathers with respect to phonons (by means of the 

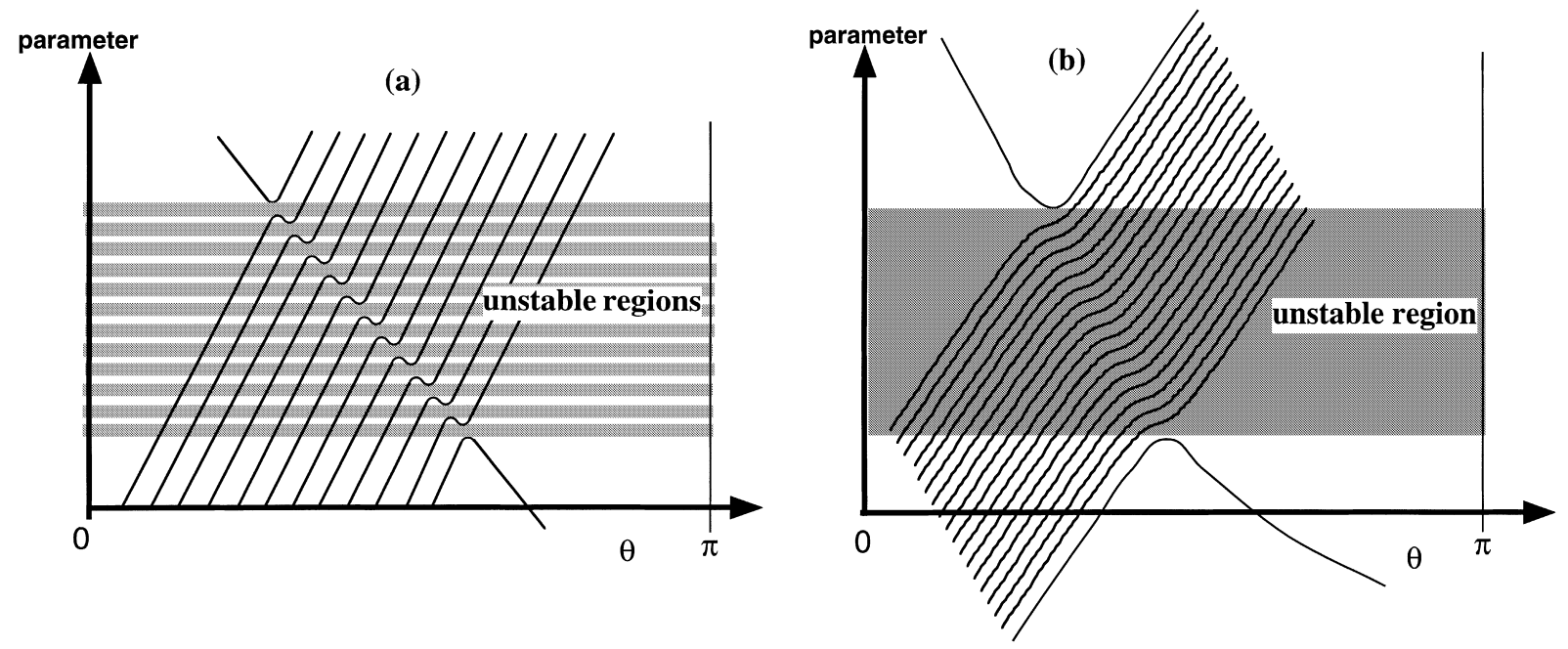

Fig. 6. Scheme of the variation of the arguments of the Floquet matrix vs. a model parameter, when a localized mode collides with the discrete phonon bands with different Krein signatures. Sequence of re-entrant instability and stability thresholds for a small system (a) or for a large system (b). The shaded areas correspond to the instability regions resulting from the Krein crunches and inverse crunches.

phase-shift). In particular the collision of localized modes with the phonon spectrum has dramatic consequences in the transmission coefficient, as explored in [1].

\subsection{Application of the band analysis}

Now we are ready to explain in detail the stability analysis of our cubic KG model, as obtained in Figs. 3-5. Since in this case the single-oscillator band is very flat and makes it hard to visualize the evolution of the crossings, we have sketched the main features of the band analysis in the schemes of Fig. 7. By "single-oscillator band" we refer to the one associated to the central oscillator of the one-site breather, which is necessarily anchored at 0,0 because it corresponds to the phase-mode present in all autonomous Hamiltonian systems.

The scheme of Fig. 7(a) shows the band analysis diagram for a much lower coupling $C$ than the one used in Figs. 4 and 5. Here we observe how the localized band associated with the central oscillator collides with the extended bands (point 1), producing an instability gap which has been shaded in gray. The scheme 7(a) is using only $N=7$ lattice sites but obviously tries to describe the features of a large system, rid of size effects.

However, in our example, this instability region is not relevant for the stability of the original breather: the only possibility for this region to extend below the line $E=0$ would be by causing a tangent (or "steady-state") bifurcation, by means of point 1 of the scheme approaching 0,0 . This is so because this localized band is anchored at 0,0 . While such tangent bifurcation is possible and has been found in several models [8], this is not the case in the cubic KG lattice. Here follows an interpretation of the numerical results.

First, one observes the birth of localized modes detaching from the bottom of the spectrum of phonons, a process that has been analyzed in detail in [12]. In the scheme, this corresponds to a band being repelled from the continuum of extended bands: it is the band which is "hybridized" with the phase-mode band above $E=0$, at point 1. Because this band is associated now to localized eigenmodes, it is capable of producing instabilities when crossing the overlapped bands, at point 2 in the scheme, much in the same way as the previous instability zone discussed above. It is this zone that becomes relevant in our example, 

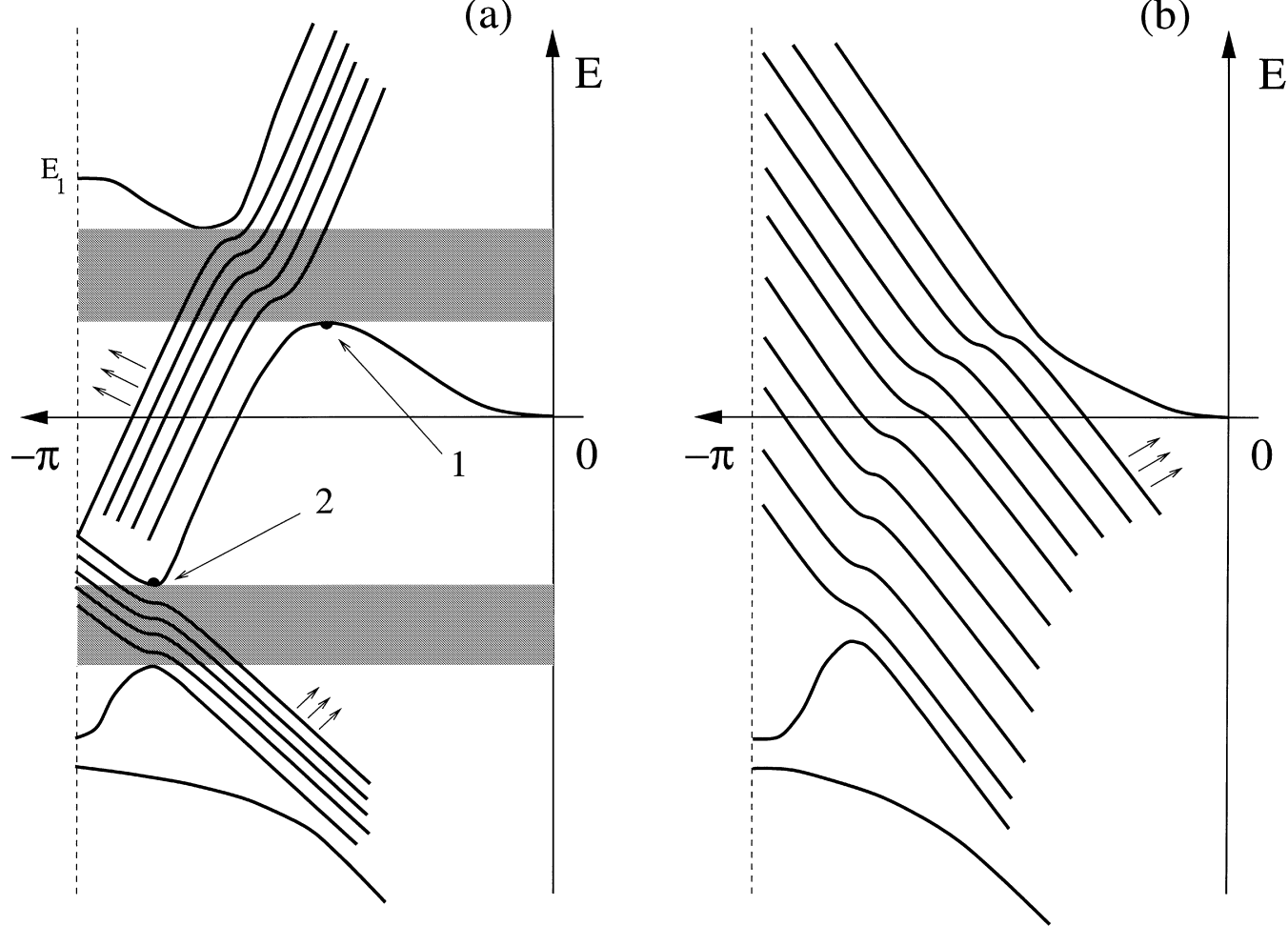

Fig. 7. Schematic interpretation of the band analysis of Figs. 4 and 5: (a) the structure at low coupling C; (b) the structure at the same coupling used in those figures, $C=0.25$ (see text).

because at some larger coupling the bands move (as depicted by the 3 little arrows), and point 2 eventually reaches the line $E=0$. In the Floquet unit circle, this means that the leading edge of the phonon bands have overlapped through -1 and caught the detached localized mode, which has not had the time to reach +1 . At that point we have a true breather instability, the one that shows up clearly in Fig. 3. At some larger coupling, points 2 and 1 of the diagram have met and the band analysis looks as depicted in Fig. 7(b), which corresponds to the zoomed view in Fig. 5. In this second scheme we have added six more lattice sites (now $N=13$ ) to make the ghost path of the localized band through the extended bands more apparent, as in the original numerical data.

Similar considerations could be made for the second localized band detached from the continuum, which was shown clearly in Fig. 4 in a stage at which finite size effects were still present. However, for simplicity in the exposition, we restricted our schematic drawings to just the first localized mode.

\section{Summary and conclusions}

In summary, we briefly reported here on several aspects of finite size effects which may appear in studies of linear stability of discrete breathers. We presented some numerical calculations and sketched their interpretation, which yielded the following conclusions valid for an infinite system:

- The collision on the unit circle of two arcs of the continuous phonon spectrum of eigenvalues of the Floquet matrix does not generate a breather instability, even when they have different Krein signature.

- The collision on the unit circle of a localized eigenvalue with the continuous phonon spectrum of eigenvalues, with different Krein signature, does (generically) create a breather instability. 


\section{Acknowledgements}

We acknowledge Thierry Cretegny for valuable discussions and comments. This work has been supported in part by the European Union, HCM program ERBCHRX-CT93-0331, and by project no. PB95-0797 of the DGICYT (Spain). One of us (JLM) acknowledges the hospitality of Laboratoire Léon Brillouin in Saclay for several visits, as well as the EU network on Stability and Universality in Classical Mechanics for support during the completion of the article.

\section{References}

[1] T. Cretegny, S. Aubry, S. Flach, Physica D 119 (1998) 73-87.

[2] A.J. Sievers, S. Takeno, Phys. Rev. Lett. 61 (1988) 970.

[3] S. Flach, C.R. Willis, Phys. Rep. 295 (1998) 181-246.

[4] R.S. MacKay, S. Aubry, Nonlinearity 7 (1994) 1623-1643.

[5] J.L. Marín, S. Aubry, Nonlinearity 9 (1996) 1501-1528.
[6] V.I. Arnold, Mathematical Methods of Classical Mechanics 2nd ed., Springer, Berlin, 1989.

[7] J.L. Marín, S. Aubry, L.M. Floría, in: G.P. Tsironis, E.N. Economou (Eds.), Proceedings of Conference on Fluctuations, Nonlinearity and Disorder, Heraklion, Greece, 1996; Physica D, to appear.

[8] J.L. Marín, Ph.D. Thesis, University of Zaragoza (1997); available at http://wanda.unizar.es /marin/PhDthesis, and upon request from the author.

[9] S. Aubry, Physica D 103 (1997) 201-250.

[10] D.K. Campbell, M. Peyrard, in: D.K. Campbell (Ed.), Chaos/Xaoc: Soviet-American Perspectives on Nonlinear Science, AIP, New York (1990), pp. 305-334; M. Peyrard, Physica D 119 (1998) 184-199.

[11] D.K. Campbell, M. Peyrard, private communication.

[12] C. Baesens, S. Kim, R.S. MacKay, Localised modes on coherent structures, Physica D (1998), these proceedings.

[13] M. Johansson, S. Aubry, Nonlinearity 10 (1997) $1151-1178$

[14] C. Kittel, Introduction to Solid State Physics, 7th ed., Wiley, 1996; N.W. Ashcroft, N.D. Mermin, Solid State Physics, Saunders College, Philadelphia, 1976.

[15] W. Magnus, S. Winkler, Hill's Equation, Wiley, Interscience, New York, 1966. 